\title{
Cyclic Approach to Web Based Project Management
}

\author{
Srinivasan Nagaraj \\ Asst. Professor \\ Dept. of CSE, GMRIT \\ Rajam - 532127, AP, India
}

\author{
M Ramachandra \\ Asst. Professor \\ Dept. of CSE, GMRIT \\ Rajam - 532127, AP, India
}

\author{
J Ratna Kumar \\ Asst. Professor \\ Dept. of CSE, GMRIT \\ Rajam - 532127, AP, India
}

\begin{abstract}
Todays world timely product delivery is much needed in order to satisfy the customer requirement as part of that well designed project management is placing important role. After being part of a new team that works with outsourcing team with dynamic allocation of resources (developers) without using a software to plan and schedule the project, web-based software model for project management. Frequently, users of project management software are not actually managing a discrete project. This paper presents project management software a New type of software based on cyclic method that are challenging the traditional definition of Project Management. Project management is the discipline of planning, organizing, securing and managing resources to bring about the successful completion of specific project goals and objectives. It is sometimes conflated with program management, however technically a program is actually a higher level construct: a group of related and somehow interdependent projects. The primary challenge of project management is to achieve all of the project goals and objectives while honoring the preconceived project constraints. Typical constraints are scope, time, and budget.The secondary - and more ambitious - challenge is to optimize the allocation and integration of inputs necessary to meet predefined objectives.
\end{abstract}

Keywords: Scheduling,Egroupware,collobrative, preconditions, critical path.

\section{INTRODUCTION}

A project is a temporary endeavor, having a defined beginning and end (usually constrained by date, but can be by funding or deliverables), undertaken to meet unique goals and objectives, usually to bring about beneficial change or added value. The temporary nature of projects stands in contrast to business as usual (or operations), which are repetitive, permanent or semipermanent functional work to produce products or services. In practice, the management of these two systems is often found to be quite different, and as such requires the development of distinct technical skills and the adoption of separate management.

\subsection{The Six Phases of Project Management}

This chapter provides a sketch of the traditional method of project management. The model that is discussed here forms the basis for all methods of project management. Dividing a project into phases makes it possible to lead it in the best possible direction. Through this organization into phases, the total work load of a project is divided into smaller components, thus making it easier to monitor. The following paragraphs describe a phasing model that has been useful in practice. It includes six phases:

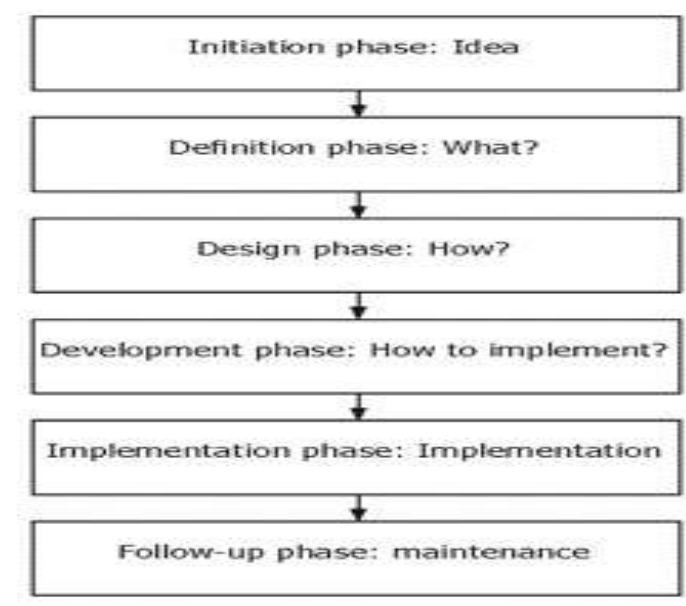

Figure 1: Project management in six phases

\subsubsection{Initiation Phase}

The initiation phase is the beginning of the project. In this phase, the idea for the project is explored and elaborated. The goal of this phase is to examine the feasibility of the project. In addition, decisions are made concerning who is to carry out the project, which party (or parties) will be involved and whether the project has an adequate base of support among those who are involved. In this phase, the current or prospective project leader writes a proposal, which contains a description of the above-mentioned matters. Examples of this type of project proposal include business plans and grant applications. In the initiation phase, the project partners enter a (temporary) relationship with each other. To prevent the development of false expectations concerning the results of the project, it makes sense to explicitly agree on the type of project that is being started: a research and development project; a project that will deliver a prototype or 'proof of concept'; a project that will deliver a working product. The choice for a particular type of project largely determines its results.

\subsubsection{Definition Phase}

After the project plan (which was developed in the initiation phase) has been approved, the project enters the second phase: the definition phase. In this phase, the requirements that are associated with a project result are specified as clearly as possible. This involves identifying the expectations that all of the involved parties have with regard to the project result. How many files are to be archived? Should the metadata conform to the Data Documentation Initiative format, or will the Dublin Core (DC) format suffice May files be deposited in their original format, or will only those that conform to the Preferred Standards be accepted must the depositor of a dataset ensure that it has been processed adequately in the archive, or is this the responsibility of the archivist? 


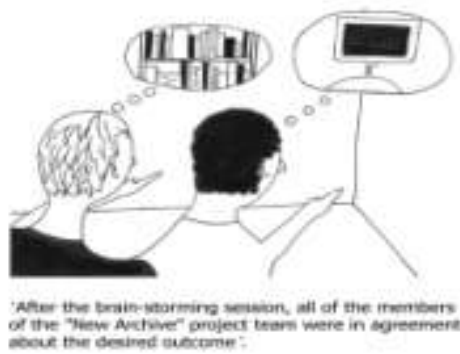

Figure 2: Expectations of a project (Illustration: Rachèl Harmsen)

It is important to identify the requirements as early in the process as possible. Wijnen (2004) distinguishes several categories of project requirements that can serve as a memory aid:

Preconditions form the context within which the project must be conducted. Examples include legislation, working-condition regulations and approval requirements.

Functional requirements are requirements that have to do with the quality of the project result Operational requirements involve the use of the project result. For example, after a software project has been realized, the number of malfunctions that occur must be reduced by ninety per cent.

Design limitations are requirements that involve the actual realization of the project. During the definition phase of a project that involved developing a web application for a consortium of large organizations, no agreements were made concerning the browser that would be supported by the application. The fact that end users are often not the ones that order the project perhaps explains why they are often ignored. The client, who pays for the project, is indeed invited to collaborate on the requirements during the definition phase.

\subsubsection{Design Phase}

The list of requirements that is developed in the definition phase can be used to make design choices. In the design phase, one or more designs are developed, with which the project result can apparently be achieved. Depending on the subject of the project, the products of the design phase can include dioramas, sketches, flow charts, site trees, HTML screen designs, prototypes, photo impressions and UML schemas. The project supervisors use these designs to choose the definitive design that will be produced in the project. This is followed by the development phase. As in the definition phase, once the design has been chosen, it cannot be changed in a later stage of the project.

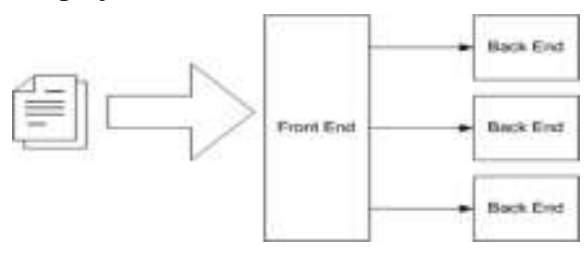

Figure 3: Example: Global design for the DANS Architecture Archive

One project involved producing a number of designs, which were quite important to the success of the project. A young designer on the project team created the designs. Although the head of the design department had ultimate responsibility for the designs, he never attended the meetings of the project team when the designs were to be discussed.

\subsubsection{Development Phase}

During the development phase, everything that will be needed to implement the project is arranged. Potential suppliers or subcontractors are brought in, a schedule is made, materials and tools are ordered and instructions are given to the personnel and so forth. The development phase is complete when implementation is ready to start. All matters must be clear for the parties that will carry out the implementation. In some projects, particularly smaller ones, a formal development phase is probably not necessary.

\subsubsection{Implementation Phase}

The project takes shape during the implementation phase. This phase involves the construction of the actual project result. It is during this phase that the project becomes visible to outsiders, to whom it may appear that the project has just begun. The implementation phase is the doing phase, and it is important to maintain the momentum. At the end of the implementation phase, the result is evaluated according to the list of requirements that was created in the definition phase. It is also evaluated according to the designs. It may be determined whether the trim on the building has been made according to the agreement that had been specified in the definition phase. This phase is complete when all of the requirements have been met and when the result corresponds to the design.

\subsubsection{Follow - up Phase}

Although it is extremely important, the follow-up phase is often neglected. During this phase, everything is arranged that is necessary to bring the project to a successful completion. Examples of activities in the follow-up phase include writing handbooks, providing instruction and training for users, setting up a help desk, maintaining the result, evaluating the project itself, writing the project report, holding a party to celebrate the result that has been achieved, transferring to the directors and dismantling the project team. Whether any results would be produced at all.

\section{CYCLICAL METHODS OF PROJECT MANAGMENT (ELIMINATING WATERFALL DRAWBACK)}

Because of the issues that have been sketched above, a number of other methods of project management have emerged in recent years. These methods are particularly suited for ITdevelopment projects. Although the above-mentioned methods of project management differ according to a number of aspects, they are essentially the same. Because the path toward the final goal of IT projects has proved so uncertain, these methods assume that the goal will be achieved in a number of short cycles. This is the background for the term cyclical project management for these methods.

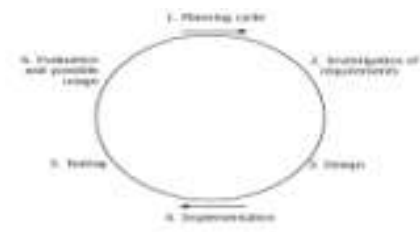

Figure 4: The Activities in a cycle 
In cyclical project management, the project goal is pursued in several short, successive consecutive cycles. Each cycle is relatively short, preferably lasting less than one month. Within each cycle, a portion of the project is carried out. Analysis, design, implementation and testing occur within each cycle. This is fundamentally different from the waterfall method, in which these activities all take place within their own separate phases. In addition, the waterfall method prescribes only single moments for definition, design, implementation and testing. In the cyclical method, this occurs many times in sequence. Various components of the software are implemented during the cycles, which are therefore independent of each other. Evaluation takes place after each cycle is completed. Should advancing insight lead to new of different requirements for the project, the activities of the subsequent cycles are adapted to take them into account. A cycle begins with the making or adjusting of the schedule. Next, the requirements of the result of the cycle are examined. A design is made, programmed and tested. Evaluation subsequently occurs and, in some methods, the new software is brought into use.

\subsection{The advantages of cyclical method are as follows:}

Higher product quality and improved implementation of functionalities, More realistic estimates of time and money, Project team is under less pressure, Higher quality.

In cyclical methods, the desired functionalities are implemented in several short cycles. In each cycle, a small portion of the desired functionality is not only investigated; it is designed, implemented and tested as well. Teams are thereby in state to make adjustments. If a design does not turn out to be good in practice, it becomes obvious during the cycle, thereby allowing adjustment. This way of working also allows customers to request adjustments. Another reason that cyclical project management leads to better quality is that a cycle involves intensive collaboration between customer, designers and programmers. A multi-disciplinary team jointly conceives of and implements solutions. In the waterfall method, the customer is involved primarily in the beginning, in the formulation of the requirements; thereafter, the designers make a design and then the programmers program the software. Cyclical methods of project management are particularly suited to projects in which the goal that is to be achieved cannot be clearly established beforehand, as in creative projects or research projects. Working in a number of cycles with a multidisciplinary team in which the end-users are also represented allows the team to discover the real goal of the project and how it can best be achieved. Each cycle contains a point for reflection and an opportunity for adjustment. In waterfall projects, a goal is formulated beforehand. Reflection on the original goal is less of a possibility.

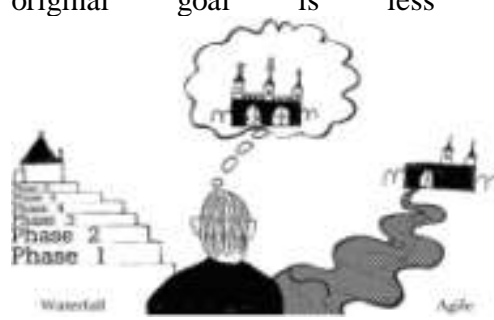

Figure 5: Better results are achieved by working in cycles. (Illustration: Rachèl Harmsen)

Quality is further strengthened by using tests as criteria for well-performing functionality from the very beginning.
Programmers must therefore define failing tests (or unit tests) before they write their code. Test-oriented work requires programmers to prove that there are no bugs in the new code before they write new code. They do this by developing a test (failing test) that would detect any possible bugs before they begin coding. The test should now succeed. The process continues in this way; for each component of the software, a test must be devised beforehand The code is not the only component that must be technically tested; the functionalities must also be tested. The prior specification of the conditions under which the new functionality can be accepted has proven particularly difficult and time-consuming. Nonetheless, the active role of customers in testing is an important determinant in the success of a project. More realistic estimation of time and money With cyclical project techniques, the functionalities that are to be implemented are not written in stone at the beginning of the project. The available time is specified. Agreements are made concerning the direction in which the project will proceed, and it is determined in the process what is actually needed, useful and realistic with regard to the software that is to be made. In cyclical projects, the functionalities that are to be implemented are not established as fixed goals, and they thus avoid the risk that the necessary hours, and therefore money, can get entirely out of hand. To prevent such a situation, the available time is used as a starting point, and it is determined during the process what is realistic to expect in that amount of time. Cyclical methods of project management are much friendlier for the project team. The team does what it can do within the specified time, but is not pressured to meet unrealistic schedules or to work within an inadequate budget. Cyclical methods also facilitate the management of external suppliers. In the cyclical working method, is theoretically possible to make new agreements for each cycle or even for each component to be delivered and, if necessary, to change suppliers.

\subsubsection{Conditions for Cyclical Project Management}

To apply cyclical project management, a number of conditions must be met:

1. Users / customers are actively involved. In cyclical project management, the formulation of requirements, design, implementation and testing take place in each cycle. This means that many decisions must be taken in a cycle. If the software is to provide a good reflection of the wishes of the customer, the customer must participate actively in the project team. Within a project, customers are involved with determining the desired functionalities and the planning of the cycles.

2. The team is authorized to take decisions. Within a cycle, the project team must be authorized to do what they think is best. If the project team does not have this power, the cyclical model of project management will not work. If constant authorization from superiors is necessary during a cycle, this can lead to stagnation.

3. Project result (software) can be broken down into smaller parts.

With cyclical project management, parts of the project are performed in a number of cycles. This is possible only if the software that is to be created is divisible into a number of more or less separate components.

4. The requirements that are imposed by management with regard to the software are primarily global; management does not impose direct, concrete or specific requirements. One of 
the strengths of cyclical project management is that the customer, designers, programmers and any testers work closely together within the cycles. 5. The activities are intelligible for the customer. If considerable technical work that is difficult for the customer to understand must take place within a cycle, a risk emerges that the customer will not be in state to participate well in the team. In such a situation, the customer has very little to contribute to the necessary design choices.

6. It should be possible to take a step backwards. Even in cyclical project management, teams sometimes pursue paths that later prove to have been wrong. In such a case, it should be possible to take a step backwards. If a new module that is created in a cycle proves inadequate, it must be possible to resume working with the old module. This imposes demands, particularly with regard to the archival and documentation of the project materials. Concurrent Versioning System (CVS) and Subversion are two helpful tools for these tasks .

7. In addition to programming, programmers should be able to communicate with customers, and vice versa. Team members must be in state to think conceptually. Discipline is necessary in order to persevere with the work.

8. The organization in which the project takes place must also offer sufficient support for this method of working. Systems for time reporting, archiving and scheduling are necessary to support the projects. These registration systems ensure the transparency that is necessary to ensure the adequate distribution of resources across projects and time.

9. Projects should have sufficient priority, team members must be released for projects. Requiring team members to work in too many projects at the same time does not work. If an organization is insufficiently adjusted to working with projects, the flexibility of cyclical project management is likely to lead to chaos.

\section{PROJECT MANAGEMENT SOFTWARE}

Project management software is a term covering many types of software, including scheduling, cost control and budget management, resource allocation, collaboration software, communication, quality management and documentation or administration systems, which are used to deal with the complexity of large projects.

\subsection{Tasks or activities of Project Management Software}

\subsubsection{Scheduling}

One of the most common purposes is to schedule a series of events or tasks and the complexity of the schedule can vary considerably depending on how the tool is used. Some common challenges include: Events which depend on one another in different ways or dependencies ,Scheduling people to work on, and resources required by, the various tasks commonly termed resource scheduling Dealing with uncertainties in the estimates of the duration of each task.

\subsubsection{Calculating Critical Path}

In many complex schedules, there will be a critical path, or series of events that depend on each other, and whose durations directly determine the length of the whole project (see also critical chain). Some software applications (for example, Dependency Structure Matrix solutions) can highlight these tasks, which are often a good candidate for any optimization effort.

\subsubsection{Providing Information}

Project planning software can be expected to provide information to various people or stakeholders, and can be used to measure and justify the level of effort required to complete the project(s). Typical requirements might include:

Tasks lists for people, and allocation schedules for resources Overview information on how long tasks will take to complete Early warning of any risks to the project Information on workload, for planning holidays,Evidence Historical information on how projects have progressed, and in particular, how actual and planned performance are related Optimum utilization of available resource.

\subsection{Approaches to Project Management Software}

\subsubsection{Desktop}

Project management software can be implemented as a program that runs on the desktop of each user. This typically gives the most responsive and graphically-intense style of interface. Desktop applications typically store their data in a file, although some have the ability to collaborate with other users or to store their data in a central database. Desktop applications can be written to run in a heterogeneous environment of multiple operating systems, although it's unusual.

\subsubsection{Web-based}

Project management software can be implemented as a Web application, accessed through an intranet, or an extranet using a web browser. It has all the usual advantages and disadvantages of web applications: Can be accessed from any type of computer without installing software on user's computer Ease of access-control and Naturally multi-user .

\subsubsection{Personal}

A personal project management application is one used at home, typically to manage lifestyle or home projects. There is considerable overlap with single user systems, although personal project management software typically involves simpler interfaces. See also non-specialized tools below.

\subsubsection{Single User}

A single-user system is programmed with the assumption that only one person will ever need to edit the project plan at once. This may be used in small companies or ones where only a few people are involved in top-down project planning. Desktop applications generally fall into this category.

\subsubsection{Collaborative}

A collaborative system is designed to support multiple users modifying different sections of the plan at once; for example, updating the areas they personally are responsible for such that those estimates get integrated into the overall plan. Web-based tools, including extranets, generally fall into this category, but have the limitation that they can only be used when the user has live Internet access. To address this limitation, some software tools using client-server architecture provide a rich client that runs on users' desktop computer and replicate project and task information to other project team members through a central server when users connect periodically to the network. 


\subsubsection{Integrated}

An integrated system combines project management or project planning, with many other aspects of company life. For example, projects can have bug tracking issues assigned to each project, the list of project customers becomes a customer relationship management module, and each person on the project plan has their own task lists, specialised tools like SourceForge integrate project management software with source control (CVS) software and bug-tracking software, so that each piece of information can be integrated into the same system.

\subsection{Web - based Project Management}

Among the open source, web-based, here is the list of the ones that seem more interesting, with some notes. I'm making this list in order to having them read to try.

\subsubsection{Endeavor Software Project Management}

Endeavour Software Project Management is an open source solution to manage large-scale enterprise software projects in an iterative and incremental development process. The major features include support for the following .

\section{Automate your software development process}

Endeavour Application Lifecycle Management drives the creation of large-scale enterprise system solutions by providing support for iterative and incremental software development processes with special emphasis on use-case driven practices.

\section{Correlate life-cycle artifacts with development efforts}

Endeavour Application Lifecycle Management features support for Use Cases, Iterations, Project Plan, Change Requests, Defect Tracking, Test Cases, Test Plans and Tasks.

\section{Track the progress of your software development team}

Endeavour Application Lifecycle Management provides you with reports and subversion repository browsing that will allow you to easily track the progress of your software development team and support for continuous integration that will keep up to date application assembly builds ready for test and demo.

\subsubsection{EGroupware}

EGroupware is a enterprise ready groupware software for your network. It enables you to manage contacts, appointments, todos and many more for your whole business. EGroupware is a groupware server. It comes with a native web-interface which allowes to access your data from any platform all over the planet.

\subsubsection{Project.Net}

Project.nets Open Source model delivers an enterprise-class, web-based project management solution without enterpriseclass pricing. Tech-savvy users are free to download and deploy Project.net on their own.

\subsubsection{Collaborative}

Collaborative is web-based project management software. It is Open Source software and provides an alternative to proprietary tools like Basecamp. Collaborative is written in PHP and JavaScript.

\section{CONCLUSION}

This paper presents project management software a New type of software are challenging the traditional definition of Project
Management. Frequently, users of project management software are not actually managing a discrete project. For instance, managing the ongoing marketing for an alreadyreleased product is not a "project" in the traditional sense of the term; it does not involve management of discrete resources working on something with a discrete beginning/end. Groupware applications now add "project management" features that directly support this type of workflow-oriented project management. Classically-trained Project Managers may argue whether this is "sound project management." However, the end-users of such tools will refer to it as such, and the defacto definition of the term Project Management may change. May not be derived from a sound project management method. For example, displaying the Gantt chart view by default encourages users to focus on timed task scheduling too early, rather than identifying objectives, deliverables and the imposed logical progress of events (dig the trench first to put in the drain pipe). Does not make a clear distinction between the planning phase and post planning phase, leading to user confusion and frustration when the software does not behave as expected. Shortening the duration of a task when an additional human resource is assigned to it while the project is still being planned.

\section{REFERENCES}

[1] Chatfield, Carl. "A short course in project management". Microsoft. us/project/HA102354821033.aspx.

[2] The Definitive Guide to Project Management. Nokes, Sebastian. 2nd Ed.n. London (Financial Times / Prentice Hall): 2007. ISBN 9780273710974

[3] Paul C. Dinsmore et al (2005) The right projects done right! John Wiley and Sons, 2005. ISBN 0-7879-7113-8. p.35 and further.

[4] Lewis R. Ireland (2006) Project Management. McGrawHill Professional, 2006. ISBN 0-07-147160-X. p.110.

[5] Joseph Phillips (2003). PMP Project Management Professional Study Guide. McGraw-Hill Professional, 2003. ISBN 0-07-223062-2 p.354.

[6] Dennis Lock (2007) Project management (9e ed.) Gower Publishing, Ltd., 2007. ISBN 0-566-08772-3.

[7] Young-Hoon Kwak (2005). "A brief history of Project Management". In: The story of managing projects. Elias G. Carayannis et al. (9 eds), Greenwood Publishing Group, 2005. ISBN 1-56720-506-2.

[8] David I. Cleland, Roland Gareis (2006). Global project management handbook. "Chapter 1: "The evolution of project management". McGraw-Hill Professional, 2006. ISBN 0-07-146045-4

[9] MorgenWitzel (2003).Fifty key figures in management. Routledge, 2003. ISBN 0-415-36977-0. p. 96-101.

[10] Martin Stevens (2002). Project Management Pathways. APM Publishing Limited, 2002. 